Journal of Computational Acoustics, Vol. 25, No. 4 (2017) 1750025 (23 pages)

(C) The Author(s)

DOI: $10.1142 / \mathrm{S} 0218396 \mathrm{X} 17500254$

\title{
More Than Six Elements Per Wavelength: The Practical Use of Structural Finite Element Models and Their Accuracy in Comparison with Experimental Results
}

\author{
P. Langer*, ${ }^{*}$, M. Maeder*, C. Guist ${ }^{\dagger}$, M. Krause ${ }^{\ddagger}$ and S. Marburg* \\ ${ }^{*}$ Chair of Vibroacoustics of Vehicles and Machines \\ Department of Mechanical Engineering \\ Technische Universität München \\ Boltzmannstraße 15, 85748 Garching, Germany \\ ${ }^{\dagger} B M W$ Group, 80788 Munich, Germany \\ ${ }^{\ddagger} I S K O$ engineers $A G, 80807$ Munich, Germany \\ §P.Langer@tum.de
}

Received 13 May 2016

Revised 21 February 2017

Published 29 March 2017

\begin{abstract}
Choosing the right number and type of elements in modern commercial finite element tools is a challenging task. It requires a broad knowledge about the theory behind or much experience by the user. Benchmark tests are a common method to prove the element performance against analytical solutions. However, these tests often analyze the performance only for single elements. When investigating the complete mesh of an arbitrary structure, the comparison of the element's performance is quite challenging due to the lack of closed or fully converged solutions. The purpose of this paper is to show a high-precision comparison of eigenfrequencies of a real structure between experimental and numerical results in the context of an element performance check with respect to a converged solution. Additionally, the authors identify the practically relevant accuracy of simulation and experiment. Finally, the influence of accuracy with respect to the number of elements per standing structural bending wave is shown.
\end{abstract}

Keywords: Finite element method; accuracy evaluation; experimental validation; virtual prototyping; finite element modeling; experimental modal analysis; uncertainty quantification.

\section{Introduction}

Engineers utilizing the finite element method (FEM) are confronted with the choice of element type and number of elements to solve a given problem efficiently. Many different element types have been implemented over the years, for example, beam, shell, plate and solid elements. ${ }^{1}$ Koschnick ${ }^{2]}$ underlines the importance of solid elements and their broad

This is an Open Access article published by World Scientific Publishing Company. It is distributed under the terms of the Creative Commons Attribution 4.0 (CC-BY) License. Further distribution of this work is permitted, provided the original work is properly cited. 
applicability. Numerical and FEMs in acoustics are well explained in the literature! ${ }^{3 / 5}$ Due to today's high demand to reduce the costs related to the development time, FEM has become increasingly important for industrial tasks. This has been clear ever since the first investigations by Kompella and Bernhard $\frac{6}{6}$ into the variation of two structure-borne and two air-borne paths of 57 apparently identical Isuzu pickup trucks. A loudspeaker and an impact hammer were used for measuring the air-borne and structure-borne transmission paths. Hills et al! 7 have compared the measurement variability of audio-frequency response of a hatchback model with both a three-door (411 vehicles) and five-door (403 vehicles) derivative and a mid-sized family five-door car (316 vehicles). In summary, the frequency response functions (FRFs) varied by approximately $5-15 \mathrm{~dB}$ over the frequency range 0 $1000 \mathrm{~Hz}$ for the structure-borne and air-borne paths.

Nowadays, the construction and development process of a new product is often separated from the actual static and dynamic analyses. For this purpose, numerical tools have been developed to help engineers. These tools numerically solve partial differential equations (PDEs) that represent certain physical behaviors, such as structural dynamics, thermomechanics or elastoacoustics. Here, the domain is divided into elements and nodes, and these equations are solved for these nodes. The nodes are connected via elements. In the first step, the element equations are formulated and approximated utilizing the Galerkin method, for example, to build the element system matrices. In a second step, the global system matrices are compounded with the use of appropriate boundary conditions. Choosing a suitable element type and the required number of degrees of freedom (DOF) for the numerical model are crucial in terms of accuracy and computational cost. Despite the presumed meaningful results a mathematical tool can calculate, it is the responsibility of the engineer to decide whether or not the result is reliable. Hornikx et al. .8 proposed a platform for benchmark cases to compare different models with respect to accuracy and modeling effort. Benchmark cases in the fields of linear acoustics, high frequency acoustics, acoustics and vibrations and acoustics involving heterogeneous and moving fluides are shall be proposed. Within this platform, analytical, numerical and experimental techniques are considered. Marburg et al $!^{9}$ have verified numerical solutions with experimentally measured data in the field of structural-acoustic modeling in order to gain knowledge about the certain degree of accuracy despite the amount of computational effort. Cifuentes and Kalbag 10 have compared linear and quadratic tetrahedral and hexahedral elements in various structural problems and observed equivalent results in terms of both accuracy and computational time. Lee and Bathe ${ }^{11}$ have investigated the influence of distortion on the performance of isoparametric quadrilateral elements. Langer et al ${ }^{[12}$ have published a study about the effect of distorted elements in three-dimensional finite element models of simple structures.

In engineering, it is common to use a fixed number of elements per wavelength to control the accuracy of finite and boundary elements. This rule has been questioned with the observation of the pollution effect which exhibits an additional error that is observed for high frequencies, see for example Babuška et al $!^{13}$ and Ihlenburg!14 It was shown that this additional error is reduced when higher order basis functions are applied $\stackrel{15}{ } \mathrm{~A}$ recent study by Dogan et al ${ }^{[16}$ has established how to evaluate the dispersion error of a meshless boundary 
element method. It was shown by Marburg and Schneider ${ }^{17 / 18}$ that there is no indication for a pollution error in boundary element methods. However, recent results of Marburg 19 challenge this observation and will further be investigated in the near future.

This paper starts with a convergence study of elements based on the element type and the DOF, followed by investigations about other discretization parameters for finite element models. This study is focused on the number of layers across the whole wall thickness of the investigated structures, the element edge length, and the element type. Natural frequencies and mode shapes obtained from a numerical and experimental modal analysis (EMA) are compared. The types of elements are restricted to solid elements to ensure a maximum of flexibility for meshing complex structures. ABAQUS/CAE is used as the numerical tool. The element formulations are almost the same in different FE software. The reader is assured that the recommendations about finite element modeling are software independent. The test cases are a simple beam and a more complex geometry, i.e. a simplified crankcase.

A short overview of deviations in natural frequencies due to uncertainties in material geometry is provided. For measuring the FRF in the frame of an EMA, a harmonic excitation by a shaker or a loudspeaker together with a scanning laser Doppler vibrometer is utilized.

\section{Theory}

\subsection{Modal analysis}

The equations of motion for a multi-DOF (MDOF) for free vibration problems in matrix notation read

$$
[M][\ddot{q}]+[D][\dot{q}]+[K][q]=[0],
$$

where $[M]$ is the mass, $[D]$ the damping, $[K]$ the stiffness matrix, and $[q]$ the components of the generalized vector of unknowns. The dot represents the derivative with respect to time. By separating of variables such as $[q]=[\hat{q}] e^{\mu t}$, the following eigenvalue problem can be stated:

$$
\begin{aligned}
\left(\mu^{2}[M]+\mu[D]+[K]\right)[q] & =[0], \\
\left(\mu_{i}^{2}[M]+\mu_{i}[D]+[K]\right)\left[\phi_{i}\right] & =[0] .
\end{aligned}
$$

Calculating all eigenvalues $\mu$ and reinstating each $\mu_{i}$ into Eq. (3), the components of the corresponding eigenvectors $\left[\phi_{i}\right]$ can be determined. For the problem of interest, $[M]$ and $[K]$ are symmetric and positive definite. For this system, real eigenvalues and eigenvectors can be expected. By assuming that $[D]$ is negligible, the system has only complex conjugated eigenvalues $\mu_{i}^{2}$, and real eigenvectors $\left[\phi_{i}\right]$.

One method for the eigenvalue extraction of symmetric systems in the FEM is the Lanczos method, which is very well described by Newman and Pipand ${ }^{20}$ as well as by Parlett. ${ }^{211}$ For further information about eigenvalue extraction, see Wilkinson ${ }^{222}$ and Bathe et al. 23 


\section{P. Langer et al.}

\subsection{Description of elements}

It is assumed that the reader is familiar with the fundamental formulations of the FEM. Therefore, the authors refrain from including a detailed description here, but refer the reader to the literature, such as Refs. 24 27. For the following studies, the commercial FEM software ABAQUS v6.10 with the pre- and post-processor ABAQUS/CAE and the implicit solver ABAQUS/Standard were utilized. Table 1 displays the elements of interest of the ABAQUS element library that have been investigated based on discussions with representatives from the industry as well as personal experiences. The use of shell elements is not part of this paper due to the limited usability for meshing complex structures. The ABAQUS library 24 provides elements with first-order (linear) and second-order (quadratic) interpolation polynomials. The user has to decide which element type has the optimal accuracy for solving the mathematical model of the corresponding physical problem. First-order elements are essentially constant strain elements. This implies that the higher-order content, for example the stress field of a beam in bending deformation, is not resolved accurately. The element with the ending $I$ has 13 additional variables representing incompatible modes. These elements, as well as second-order elements exactly capture a linear strain field. Furthermore, the user is left with the question of whether full or reduced integration is applied for building the system matrices. All tetrahedral elements use full integration. Full integration only signifies that the number of integration points is sufficient to calculate the virtual work expression most accurately. For the reduced integration procedure, the integration points are at the locations that gain optimal accuracy. These points or locations are

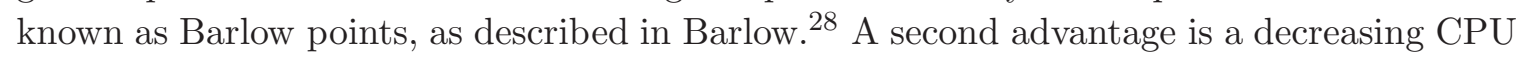

Table 1. Description of three-dimensional finite element types. 24

Name
C3D8I


time and storage requirements. The primary disadvantage is that this integration permits deformation modes that do not cause any strain field at the integration points and therefore inaccurate solutions, since only first-order quadrilateral and hexahedral elements are taken into account. The authors also use quadratic elements with reduced integration, since the fully integrated elements are not capable of resolving nearly incompressible material behavior. This drawback is known as volumetric locking. By referring to the treatises, 26|29 31 one can see that fully integrated first-order isoparametric elements can have problems with bending of thin structures due to shear locking.

\section{Description of Test Specimens}

Figure 1 shows the test specimens. A beam-like structure with nominal dimensions of $(0.2 \times 0.04 \times 0.004 \mathrm{~m})$ and a simplified crankcase structure made of aluminum are investigated, for which linear elastic material behavior, i.e. Hooke's Law, is assumed. Figure 1(a) shows the beam specimen and Fig. 1(b), the investigated more, complex structure. The dimensions are comparable to a real crankcase of a three cylinder engine. All radii of the investigated structures were manufactured with less than $0.005 \mathrm{~m}$. Langer et al $l^{[12]}$ discussed the question how accurately edges and curves of the real component should be simulated. The investigated radius of $0.005 \mathrm{~m}$ on monolithic beam structures has a negligible influence

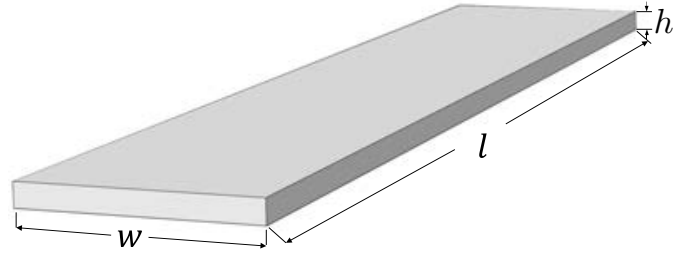

(a)

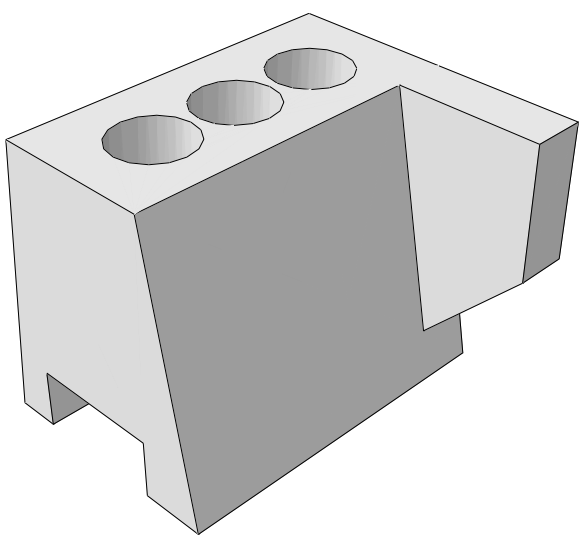

(b)

Fig. 1. Investigated structures. (a) Simple beam structure; $l=0.2 \mathrm{~m}, w=0.04 \mathrm{~m}, h=0.004 \mathrm{~m}$ and (b) more complex structure - simplified crankcase.

Table 2. Mean value of material parameters.

\begin{tabular}{lcc}
\hline Property & Value & Unit \\
\hline Density $[\varrho]$ & 2674 & $\frac{\mathrm{kg}}{\mathrm{m}^{3}}$ \\
Young's Modulus $[E]$ & 71 & $\mathrm{GPa}$ \\
Poisson's Ratio $[\nu]$ & 0.33 & - \\
\hline
\end{tabular}




\section{P. Langer et al.}

to the calculated eigenfrequencies and the accuracy with respect to experiments. The material properties have been experimentally validated and are presented in Table 2 .

\section{Experiments}

By performing an EMA, it is possible to measure the resonance frequencies of a structure. The modal parameters such as eigenfrequencies, modal damping, and eigenvectors are numerically evaluated utilizing a curve fit algorithm along with a decomposition scheme of the fitted polynomials. In this paper, only the eigenfrequencies of the examined structures are considered. For the sake of clarity in this section, the general setup of the experiments is explained, while details of the structures are presented afterwards.

\subsection{Beam setup}

For the EMA, results depend on the precision of the measuring device and the apparatus used in the experiment. Therefore, a suitable measurement technique must be carefully chosen to correctly record the excitation and the structural response. To examine the beam specimen, the structure is exited with a loudspeaker while measuring the sound pressure at a reference position. However, this method is only applicable for sound sensitive structures. A review of different measurement techniques is provided by Ewins ${ }^{32}$ as well as the International Organization for Standardization $\underline{33}$

In all tests, the boundary condition is considered as free-free for simplicity with respect to the simulations explained in the following sections. The authors understand that this is an ideal estimation of the real setup. But since the frequencies of the corresponding rigid body motions were less than 10 times the resonance frequency of the first deflection shape, the mutual influence is considered to be negligible.

In the measurement setup, the beam is attached to two elastic strings in an anechoic chamber, see Fig. 2. A microphone measures the sound pressure level in front of the loudspeaker, which excites the structure with a periodic chirp signal. The advantages of the applied signal are its continuity up to the first derivative and its periodicity within the time block. Therefore, a continuous, sequential measurement utilizing a scanning laser Doppler vibrometer is possible. The time signals of all quantities were windowed with a RectangularWindow, transformed into frequency domain with the use of the Fast Fourier Transformation (FFT) and handled further to obtain the FRFs of each measurement. Handing over the FRFs to ME'Scope 34 as a postprocessing tool, the eigenfrequencies and the associated mode shapes are identified.

\subsection{Simplified crankcase setup}

As mentioned before, the use of a loudspeaker is only applicable for structures that are sound sensitive. In the second test case, a simplified geometry of a three-cylinder crankcase is analyzed. Since this structure's impedance is too large for it to be excited by a loudspeaker, a shaker excitation together with a force transducer has been preferred. A scanning laser 


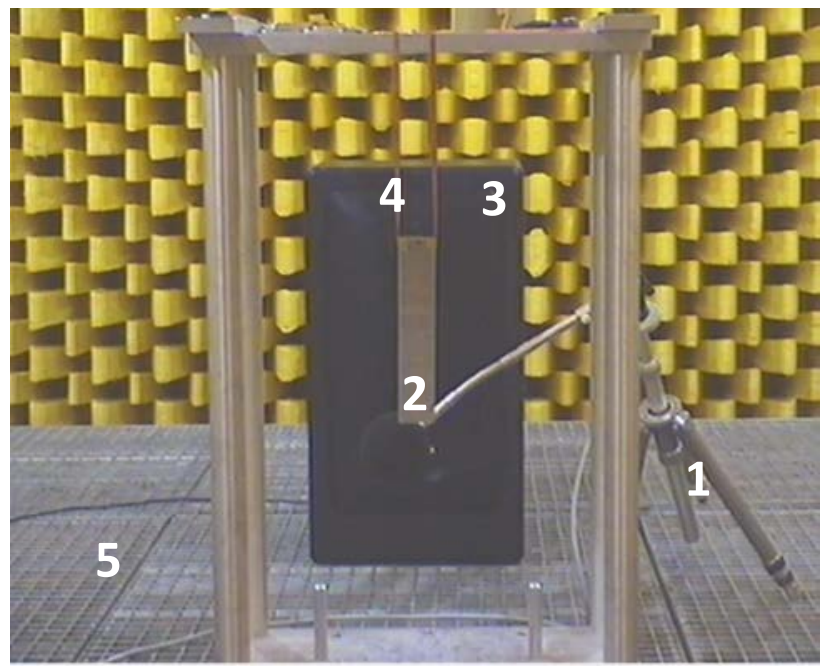

Fig. 2. Experimental setups for beam structure: (1) microphone, (2) specimen, (3) loudspeaker, (4) elastic strings, (5) anechoic chamber.

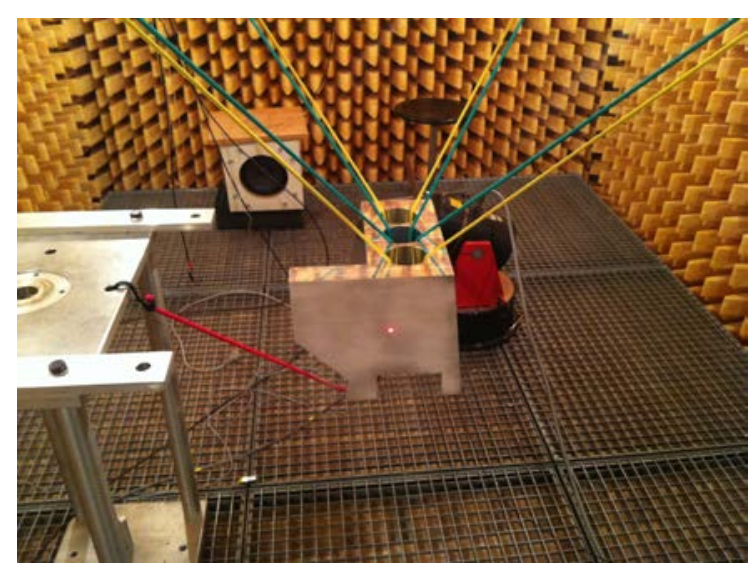

(a) Shaker position 1

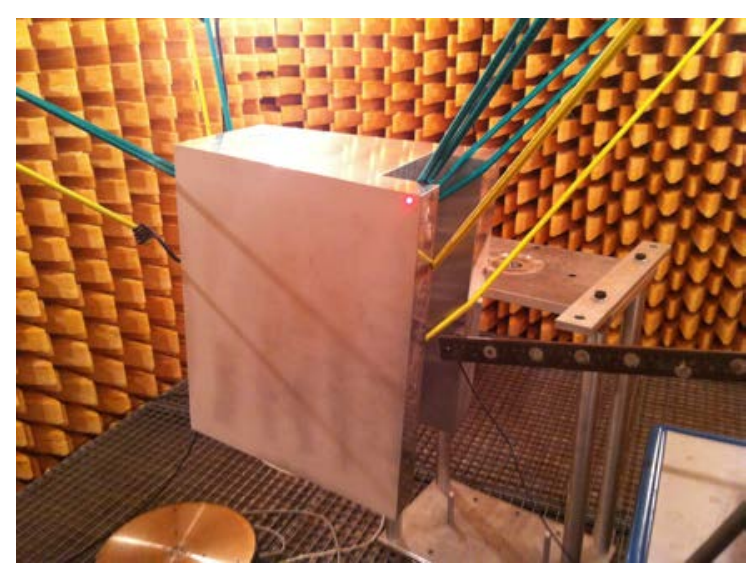

(b) Shaker position 2

Fig. 3. Experimental setups for complex structure.

Doppler vibrometer measured the surface normal velocity at discrete points on the structure. Again, a periodic chirp signal for the shaker allowed a rectangular window for transforming all measured time signals into the frequency domain.

\section{Example 1: Beam Structure}

\subsection{Model description}

Figure 4 shows the finite element model that was created using ABAQUS/CAE. The mesh consists of 640 second-order brick elements (C3D20). To minimize the numerical error, no 


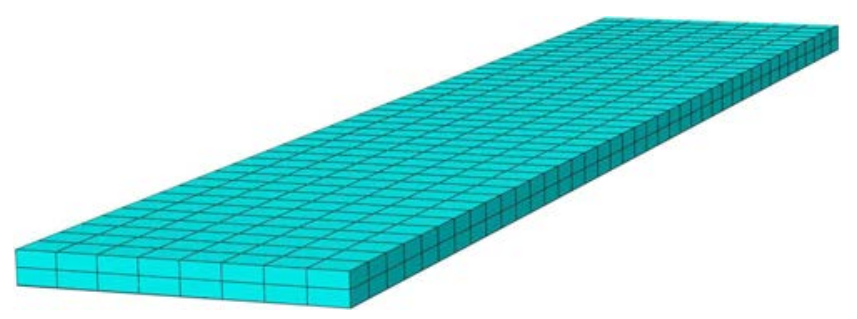

Fig. 4. Finite element model of the beam structure.

distorted elements were allowed in the process of meshing the geometry. The boundary conditions are ideal free-free. In order to compare results of simulations with experimental results, a converged solution is required. C3D20R elements tend to yield more accurate results when using fewer DOF in comparison to the other element types of interest.24|28

\subsection{Eigenfrequency error}

\subsubsection{Error measures}

A converged solution is assumed when the results, in the frame of a numerical modal analysis, vary less than $1 \%$ after doubling the DOF. Using these results, a relative error can be calculated:

$$
\varepsilon=\left|100 \cdot\left(\frac{f}{f_{\text {con }}}-1\right)\right| \%,
$$

where $f_{\text {con }}$ is the eigenfrequency from the converged numerical calculations and $f$ is the corresponding eigenfrequency obtained by the experiment or simulation with fewer DOF or different element types than the converged reference solution. Furthermore, a comparison of the CPU times required to solve for the numerical results is presented. All calculations were executed on a 64-bit Windows machine with 16 cores @ $2.53 \mathrm{GHz}$ and 24 GByte RAM. In the following, a sufficiently accurate solution of a finite element model, where the relative deviation for the investigated eigenfrequencies is less than $1 \%$ to a reference model, is defined. This virtual model has a larger number of DOF to ensure convergence.

\subsubsection{Identify practically relevant accuracy}

A reduction of the threshold value of $1 \%$ is not useful, according to the results in Fig. 囵. The numerical and experimental results for the eigenfrequencies of the first three bending modes of 10 beam samples are presented.

The numerical models are meshed with quadratic elements C3D20 and C3D10, as well as with a mesh with lower resolution, which has been used by Langer et al .35 Experimental results are shown with black solid curves labeled "LDV". The solution of the numerical model is influenced by all input parameters and their specific uncertainties. These parameters include geometrical dimensions as well as material parameters of Hooke's model. The dimensions $l, w$ and $h$ of all samples have been measured to identify the true deviations 


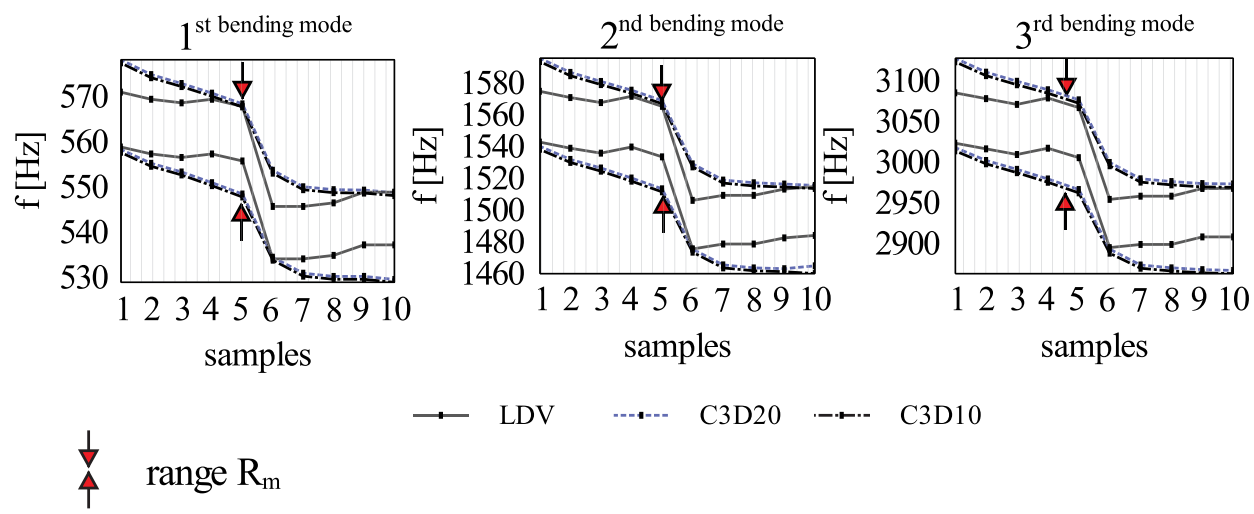

Fig. 5. Range $R_{\mathrm{m}}$ of ten finite element models of simple beam structures in the first three eigenfrequencies of bending modes due to uncertainties in material and geometry. LDV: experimental results; C3D20 and C3D10: solutions of finite element models discretized with quadratic hexahedral and tetrahedral elements.

within the set. The material parameters are Young's modulus, $E$, the density, $\varrho$ and Poisson's ratio, $\nu$. For an accurate determination of the material parameters, the pulse-echo method has been used to measure the velocity of sound of the longitudinal wave and transversal wave in each sample. With the high-precision measurement of the density $\varrho$, we can calculate the Young's modulus $E$ and Poisson's ratio $\nu$. The relevant uncertainties arise from the error propagation by taking into consideration the accuracy of all measurement data and their statistical fluctuations. For each sample, the standard deviation and measurement uncertainty are determined from the mean values. The maximum averaged deviations from the mean value are $\pm 2.3 \%$ for the Young's modulus, $\pm 0.2 \%$ for the density and $\pm 3.3 \%$ for the Poisson's ratio. The relative deviation for the shape parameters are $\pm 0.01 \%$ for the length, $\pm 0.05 \%$ for the width and $\pm 0.5 \%$ for the height. By means of the error propagation theorem, upper and lower bounds around the mean values can be stated. This indicates the range $R_{\mathrm{m}}$, representing the total uncertainty. That means that for the first three eigenfrequencies of bending modes of the simplest structure, a relative deviation surrounds the mean values of $\pm 1.75 \%, \pm 2.1 \%$ and $\pm 1.9 \%$ in the finite element solution, respectively. As a result of this considerable uncertainty, it can be assumed that a relative deviation of $1 \%<\varepsilon<2 \%$ between simulations and experiment is reasonably accurate. A lower deviation is inside the distribution of the numerical results due to material and geometry uncertainties.

In addition, it was found to be physically correct that the physical model is limited by the solution of numerical models. Figure 5 shows a perfect match between the ranges of the finite element models and the experiment.

\subsubsection{Number of elements per wavelength}

The goal of this study is to investigate the number of elements (el) per wavelength $(\lambda)$ in order to achieve a criterion for a sufficiently accurate numerical modal analysis. Figure 6 shows the structural wavelength of the beam structure investigated. The boundary condition is free-free. The first bending mode consists of a half of a wavelength $(\lambda / 2)$ and the second 
half of a wave

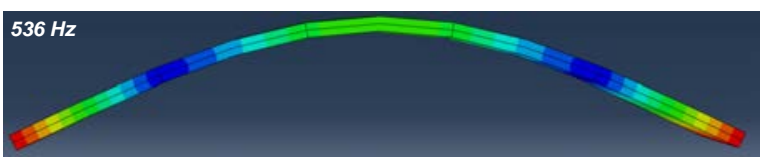

one wave

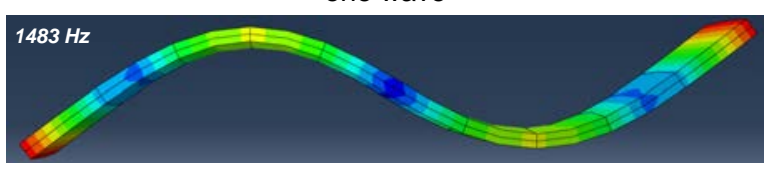

one and a half waves

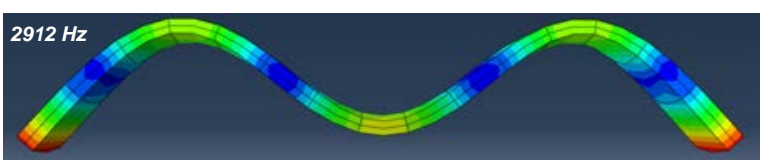

two waves

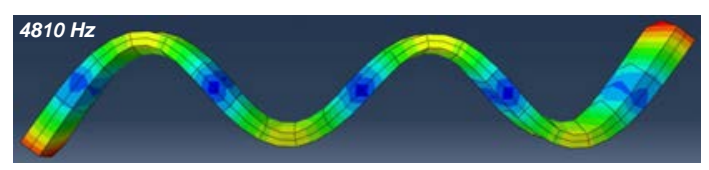

two and a half waves

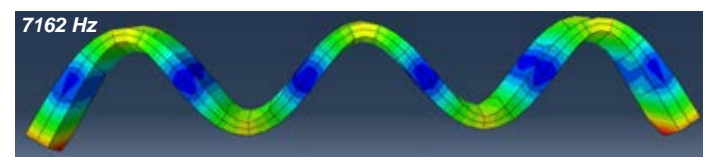

three waves

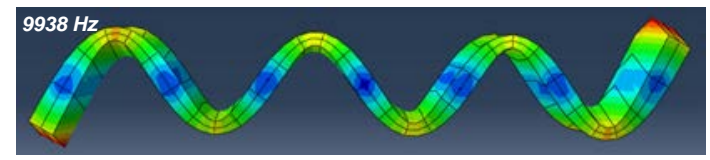

Fig. 6. Structural wavelength $(\lambda)$ of the beam structure.

mode shows one complete wave. The number of elements per wavelength were constant over the whole frequency range for the first specific investigation. The other parameters of discretization, such as the element type and the number of layers, remain unchanged. The finite element model had two layers of elements over the thickness and one layer over the width of the beam. A frequency range up to $10 \mathrm{kHz}$ was chosen, because the focus was exclusively on the first six natural frequencies of the bending modes. Two mesh settings are examined: one with elements using a quadratic formulation (C3D20) and another one using a discretization scheme with linear formulation (C3D8).

Figure 7 shows the relative error of finite element models with a fixed number of elements per wavelength for the eigenfrequencies of the first six bending modes compared to a reference model with 2000 quadratic elements across the length $l$. Due to the resulting bad shapes of these elements, the reference model was verified with a finite element model with four layers across the width. The result was a negligible influence of the model solution for using one layer across the width. The number of elements per wavelength — four, six, ten, twenty, forty, and five hundred - are taken into consideration. Using second-order elements, 20 elements per wavelength are sufficient to achieve results within a $1 \%$ error up to $10 \mathrm{kHz}$. To calculate the first natural frequency of a finite element model with $400 \mathrm{DOF}$, this value is quite sufficient. The computational time for the highest natural frequency of this model is less than $0.1 \mathrm{sec}$. For fewer elements per wavelength, higher relative errors can be expected. For finite element models discretized with linear elements, at least 500 elements per wavelength are needed to achieve a relative error below $1 \%$. This leads to a finite element model with 4500 DOF and a computational time of 0.6 sec. The error increases significantly with fewer elements per wavelength. Accuracy of finite element simulations is nearly independent of the variation of the number of elements in the width of the beam.

A further effect in the error analysis is observed. The relative error decreases with a higher number of bending modes for models discretizised with quadratic and linear elements. This phenomenon was determined independently of the number of elements per wavelength. 

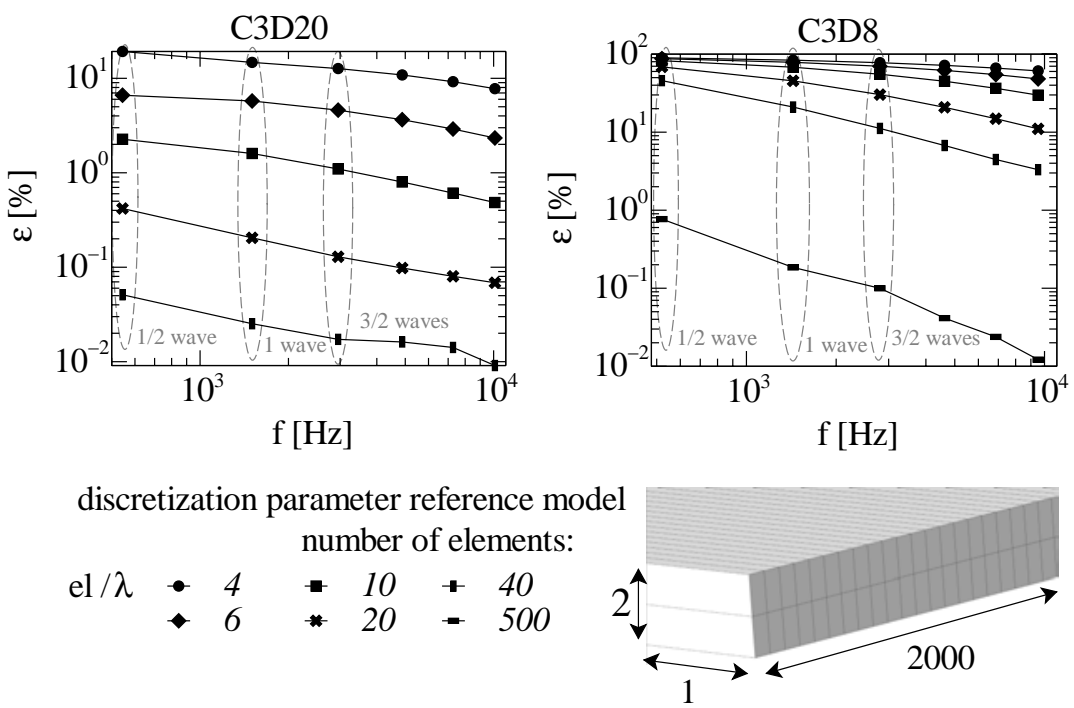

Fig. 7. Relative error with a fixed number of elements per wavelength for the eigenfrequencies of the first six bending modes.

\subsubsection{Behavior of relative errors}

Referring to the corresponding literature, see Refs. 36-40, it was assumed that the error due to the pollution effect should increase with higher wavenumbers, because the rule of using a fixed number of elements per wavelength is not sufficient for high frequencies. However, when discussing this, it should be clarified that the pollution effect is usually measured as a displacement error (structural elements) or a sound pressure error (acoustics) in the $\mathrm{H}^{1}$-norm or the $\mathrm{H}^{1}$-seminorm. Eigenfrequencies can be understood as the quotient of two energy norms (generalized Rayleigh quotient) but do not refer to the local derivative as the $\mathrm{H}^{1}$-norm does. When analyzing the error with respect to the eigenfrequencies, the pollution effect cannot be identified. The effect observed here has even been the opposite to the pollution effect. In the following, further investigations are presented to understand this phenomenon.

In order to achieve minimal influence of the element edge length, beams with quadratic cross section have been analyzed. In this case, the examined meshes are composed of cubeshaped elements. Figure 8 shows the relative error for the first four eigenfrequencies of bending modes to a reference finite element model with 2500 quadratic elements across the length $l$. Contrary to the expectations for quadratic and linear elements, the relative error decreases again with higher eigenfrequencies of bending modes.

To investigate not only finite element models with elastodynamic material behavior, an acoustic model was considered by solving the Helmholtz equation. Therefore, a further examination is to solve a one-dimensional duct problem. In this case, it is the solution of the Helmholtz equation for an air-filled duct with the length $l=1 \mathrm{~m}$. The material data of air consists of the density $\varrho_{\text {air }}=1.3 \mathrm{~kg} / \mathrm{m}^{3}$ and the speed of sound $c_{\text {air }}=340 \mathrm{~m} / \mathrm{s} \stackrel{41}{\text { Figure }} 9$ 

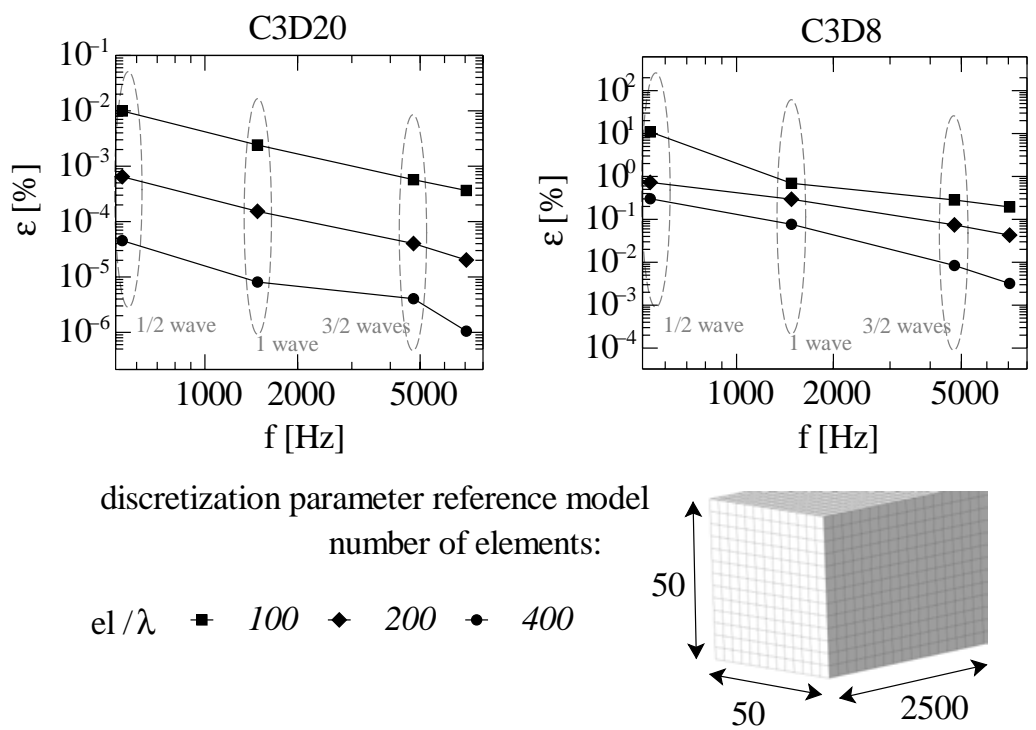

Fig. 8. Beam with the length $l=0.2 \mathrm{~m}$ and quadratic cross section area $(0.2 \times 0.004 \times 0.004 \mathrm{~m})$ : Relative error with a fixed number of elements per wavelength for the first four eigenfrequencies of bending modes.

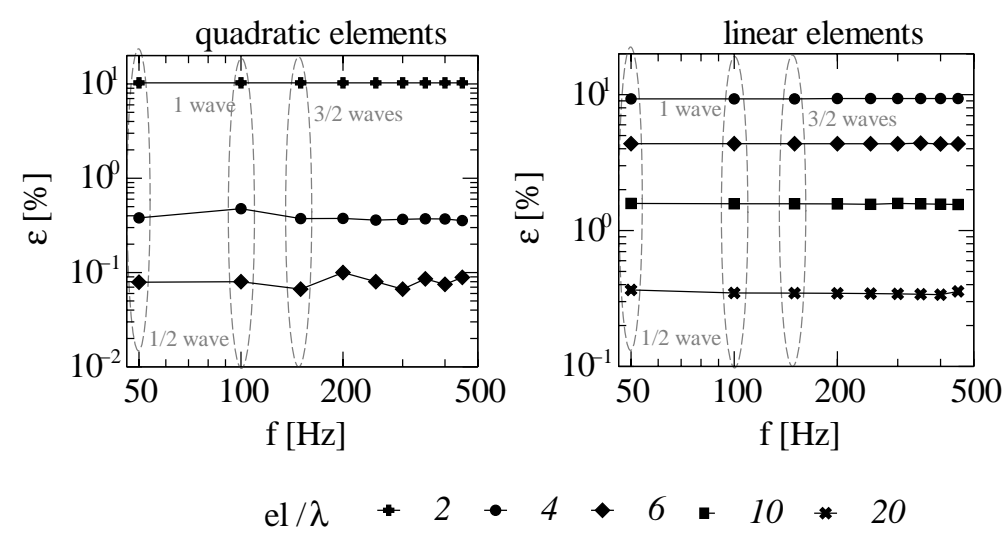

Fig. 9. One-dimensional duct: Relative error with a fixed number of elements per wavelength to a reference model for the first nine eigenfrequencies.

shows the relative error with a fixed number of elements per wavelength to a reference model for the first nine eigenfrequencies using quadratic and linear elements. Regarding the relative error, a highly accurate solution is reached, with six quadratic elements per wavelength and a relative error of less than $1 \%$ to a reference model with 500 quadratic elements per wavelength. The model meshed with linear elements provides high quality solutions with more than 10 elements per wavelength. It can be found that the relative error in models with quadratic and linear elements is constant over the whole frequency range. This is in strong contrast to the results of the three-dimensional structural finite element models. 
The presented results are in contrast to the investigations by Oberai and Pinsky, 12 who discussed the effect to pollution through the comparison of FEMs for the Helmholtz equation. The authors explain this with the properties of the directions normal to the longitudinal axis of the three-dimensional beams: In a bending deflection, the shear stress $\tau_{12}$, where the 1-direction is along the length and the 2-direction is along the height of the beam, has a quadratic progression between the upper and the lower side faces. According to this, the accuracy of this approximation is higher with more elements in the transverse direction.

\subsubsection{Discretization parameters}

In relation to global discretization parameters, note the following statements for thin-walled structures: Not only the number of DOF and the element formulation are essential for highly accurate solutions of finite element models; in order to achieve an accurate numerical solution for thin-walled structures, initial studies have shown that the discretization parameters, such as the number of layers across the thickness $l_{n}$ and the regularity of the mesh, are essential as well. Of course, with increasing the number of layers and the use of a shorter element edge length, DOF increase in the finite element model as well. The using of a regular mesh in finite element models is recommended. That can generate positive effects, such as less computational time with constant accuracy due to optimally shaped elements. Figure 10 shows the deviation of different mesh strategies compared to a reference model. The element edge length, the element type, and the number of layers have been varied. These parameters are altered in such a way that no distorted elements are generated. Therefore, for element edge lengths of $5 \mathrm{~mm}, 1 \mathrm{~mm}$ and $0.5 \mathrm{~mm}$, it has been possible to generate 1,2 , or 3 layers over the thickness.

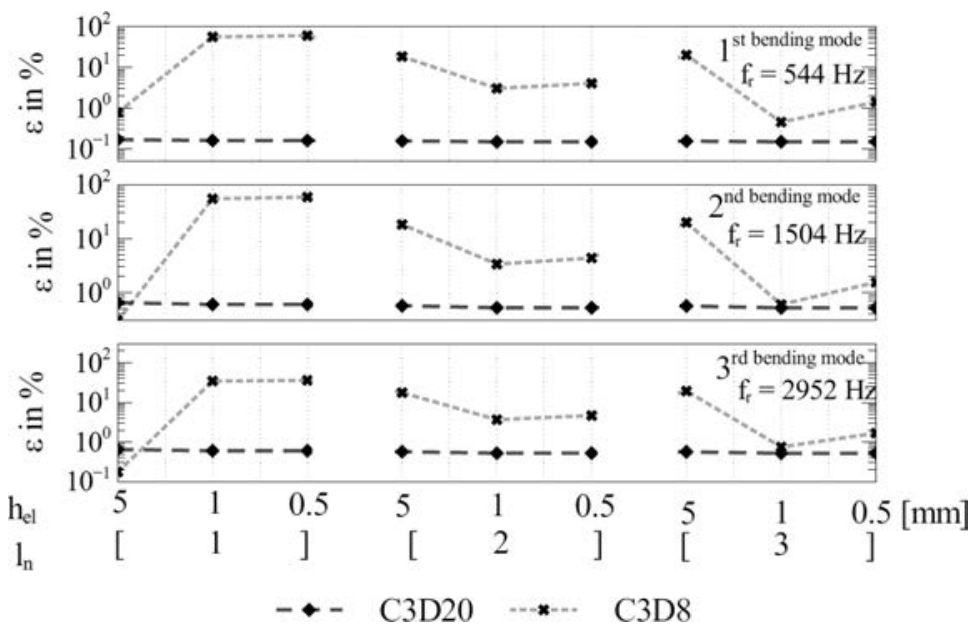

Fig. 10. Relative deviation of one finite element model with different discretizations to a reference model for the first three eigenfrequencies of bending modes. $h_{\mathrm{el}}$ : element edge length in mm; $l_{n}$ : number of layers across the thickness $h ; f_{\mathrm{r}}$ : approximate solution of the reference model. 


\section{P. Langer et al.}

The eigenfrequencies $f_{\mathrm{r}}$ are the results of the reference finite element model with quadratic elements, $0.5 \mathrm{~mm}$ element edge length, and $3.4 \cdot 10^{6}$ DOF. In general, apart from other discretization parameters, the solutions of finite element models meshed with second-order elements demonstrate a more stable solution. Compared to a mesh with linear elements, here, a good conditioning of the element shape, close to the cubic form, is less essential. For example, despite the increased number of DOF, a mesh with an element edge length of $5 \mathrm{~mm}$ and one layer yields more accurate results compared to a discretization with 1-mm element edge length and one layer. In the latter case, the elements aspect ratio is too large and the element shape resembles a stretched rectangle. That means that for linear elements, a fully converged solution cannot be achieved. This is also observed for meshes with more layers over a 1-mm element edge length. These high deviations can arise due to a variety of reasons, e.g. a not completely converged solution, or a mesh which tends to a numerical solution includes stiffening effects, called locking effect. Koschnick ${ }^{2}$ describes geometric and material stiffening effects from three different points of view: the numerical, the mathematical, and the mechanical. According to this description, a numerical convergence to the exact solution can occur with larger DOF, but the computation time is much longer compared to locking-free elements. In order to understand different locking effects, a critical parameter can be defined to describe the influence on the solutions of virtual models. For the dimensions of the investigated beam structures, geometrical stiffening effects can additionally be taken into account. One of these effects is shear locking for bending structures. Here, the critical parameter is the aspect ratio of the numerical elements. A good explanation is provided by Sun $\frac{43}{4}$ and Belytschko. $\frac{44}{4}$ A more detailed process of analyzing the reasons and evaluations of stiffening effects can be found in the literature, such as Babuška and Suri 45

\subsubsection{Guideline for thin-walled structures}

These results provide a clear indication that the dicretization parameters, such as the number of layers across the thickness $l_{n}$, the number of elements per wavelength el $/ \lambda$, and the elements aspect ratio $\alpha_{\mathrm{el}}$, play a role as well. With the experience gained from several studies of element types, $\sqrt{35}$ investigations of torsion modes, as well as the variation of many discretization parameters, a recommendation:

Table 3 shows a guideline for a meshing process of three-dimensional finite element models for thin-walled structures, sufficient for an accurate solution up to the mid-frequency range. The guideline's data indicates an accuracy of $1 \%$ of the calculated eigenfrequencies to a reference finite element model with a larger number of DOF. For an accurate finite element model to analyze eigenfrequencies of bending modes, two and three layers across the thickness $l_{n}$ are sufficient for quadratic and linear elements, respectively. For the highest considered bending mode, efficient discretizations for linear and quadratic element formulation are 500 and 20 elements per wavelength, respectively, with a maximum element's aspect ratio of $1: 4$ and 1:10. That means that for the mesh with linear element formulation, many more DOF are required. 
Table 3. Guideline for discretization parameter for meshing thin-walled structures. $p$ : order of basis function; el $/ \lambda$ : minimum number of elements (el) per wavelength $(\lambda)$ for bending mode; $l_{n}$ : minimum number of layers across the thickness; $\alpha_{\mathrm{el}}$ : elements aspect ratio.

\begin{tabular}{lcc}
\hline & \multicolumn{2}{c}{ Bending Mode } \\
\cline { 2 - 3 }$p$ & Linear & Quadratic \\
\hline $\mathrm{el} / \lambda$ & 500 & 20 \\
$l_{n}$ & 3 & 2 \\
$\alpha_{\mathrm{el}}$ & $1: 4$ & $1: 10$ \\
\hline
\end{tabular}

\subsubsection{Error of natural frequency in terms of DOF}

Figures 111 and 12 display the results for the first bending and lateral modes of the beam. These investigations are independent of the discretization parameter in the finite element models and focus only on the DOF. Since it is not possible to measure lateral deflection with the previously described experiments, Fig. 12 does not show experimental results. The discretizations with results with less than a $1 \%$ relative error are useful for high-quality statements.

For the bending deflection in Fig. 11, the experimental results using $2 \cdot 10^{5}$ C3D20R elements differ about $0.2 \%$ from the converged solution. This element type provides highest accuracy with the lowest number of DOF. Linear and quadratic elements converge from

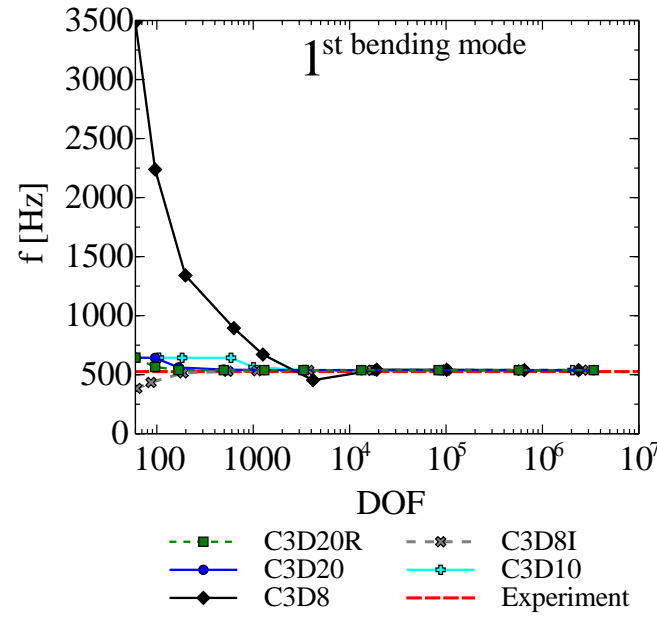

(a)

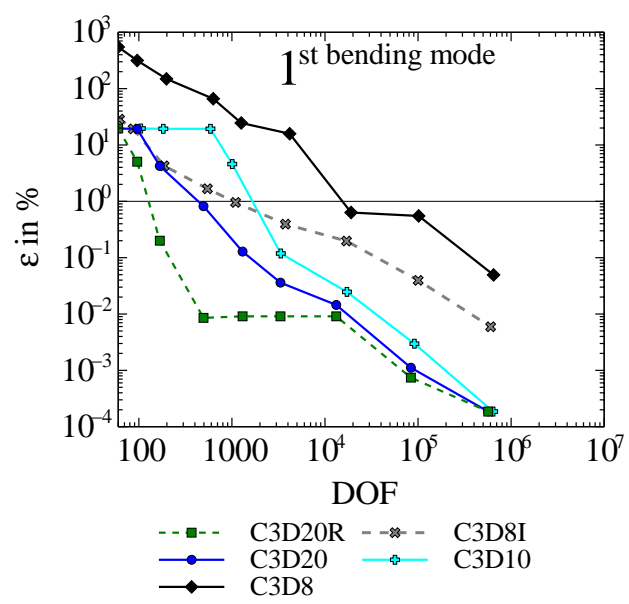

(b)

Fig. 11. Beam results - bending mode. (a) Eigenfrequency of first bending mode from finite element models with different types of elements and experiment and (b) relative error of first bending mode to a reference finite element model with $3.4 \cdot 10^{6}$ DOF and quadratic elements. 


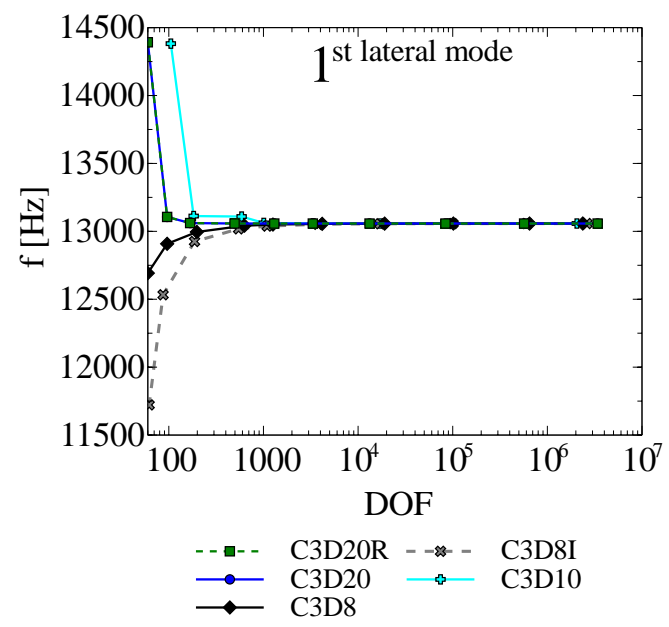

(a)

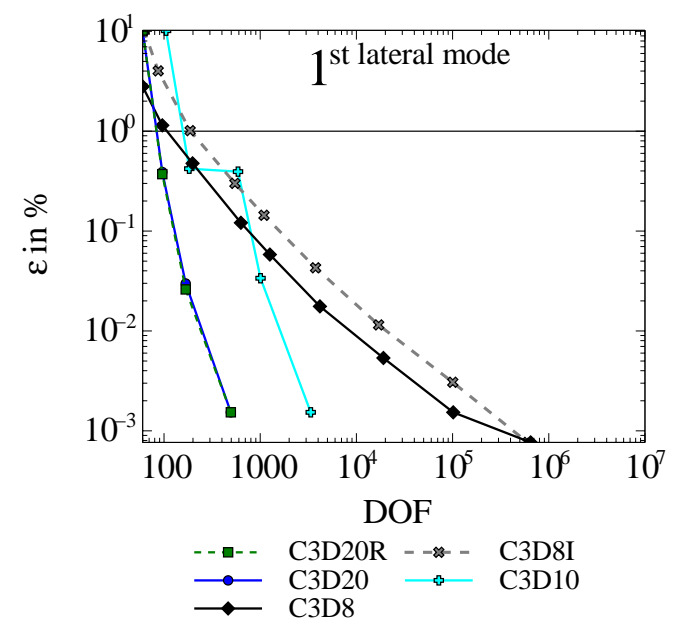

(b)

Fig. 12. Beam results - lateral mode. (a) Eigenfrequency of first lateral mode from finite element models with different types of elements and (b) relative error of first lateral mode to a reference finite element model with $3.4 \cdot 10^{6}$ DOF and quadratic elements.

above. Furthermore, it can be noted that quadratic elements tend to yield more accurate results than linear elements with respect to constant DOF. With quadratic elements, the same quality as with the use of linear elements can be achieved using a number of DOF ten times less. The model with C3D8I elements has more accuracy than the C3D8 models with the same number of DOF. This is only the case for a regular mesh. Additionally, the C3D8I model produces better results with fewer number of DOF. When the elements deform

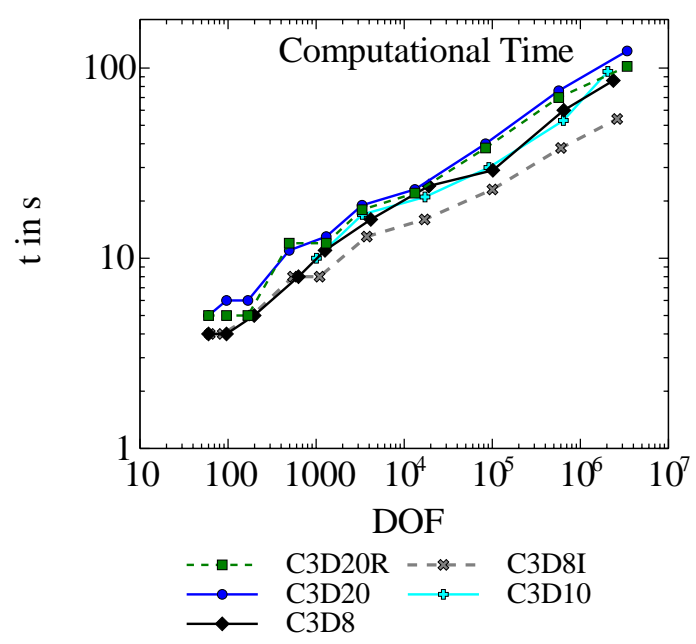

Fig. 13. Comparison of computational time (beam) of the finite element models with different types of elements. 
laterally, the results are quite smooth, since originally finite elements are good to solve for a homogenous stress distribution within the element. Missing values in Fig. 12 indicate that there is no difference between the converged solution and the solutions obtained with fewer elements or with different types of elements in logarithmic scale. Linear elements converge from below, quadratic elements from above.

\subsubsection{Computational time in terms of DOF}

Taking into account the computational time in Fig. 13 needed to obtain the results, it can be observed that quadratic elements achieve more accurate results in less wall clock time. For the linear elements, the C3D8I model has a higher accuracy than the C3D8 model and requires less computational time. For these investigations, the standard eigensolver Lanczos was used.

\section{Example 2: Complex Geometry, a Simplified Crankcase}

\subsection{Model description}

The behavior of elements has been further investigated using a more complex geometry. Figure 14 shows the finite element model of a simplified crankcase. The boundary conditions are ideal free-free and no distorted elements were present.

\subsection{Error of natural frequency in terms of DOF}

Focusing on the first three calculated structural eigenfrequencies, the elements' performance is shown in Figs. $15,17$.

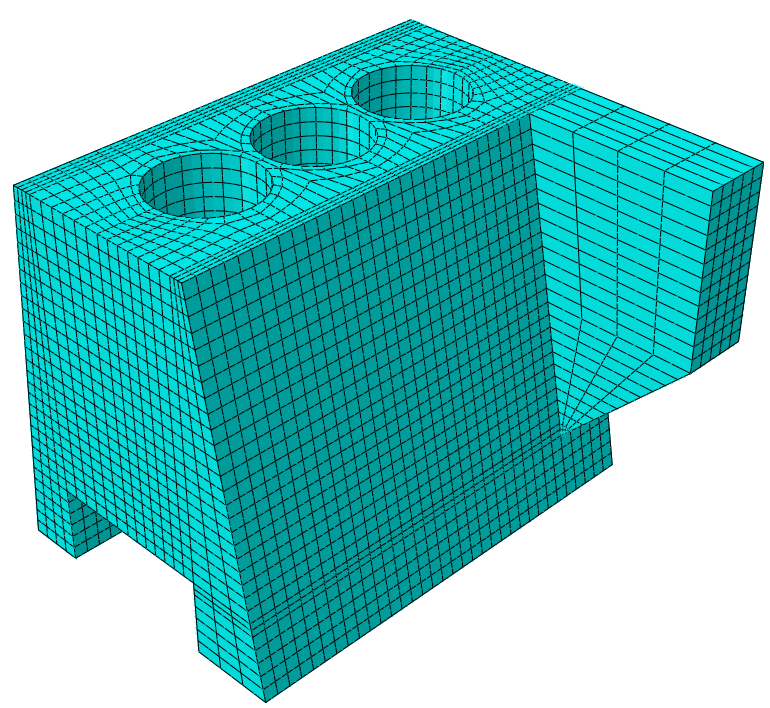

Fig. 14. Finite element model of a simplified crankcase as a more complex structure. 


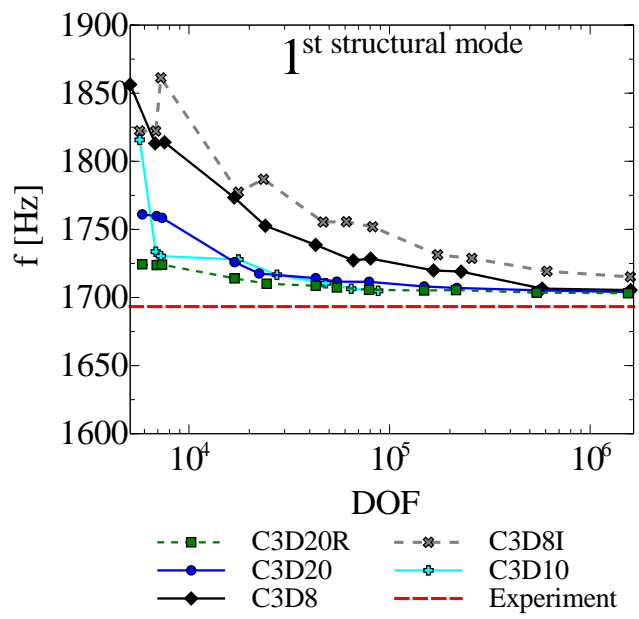

(a)

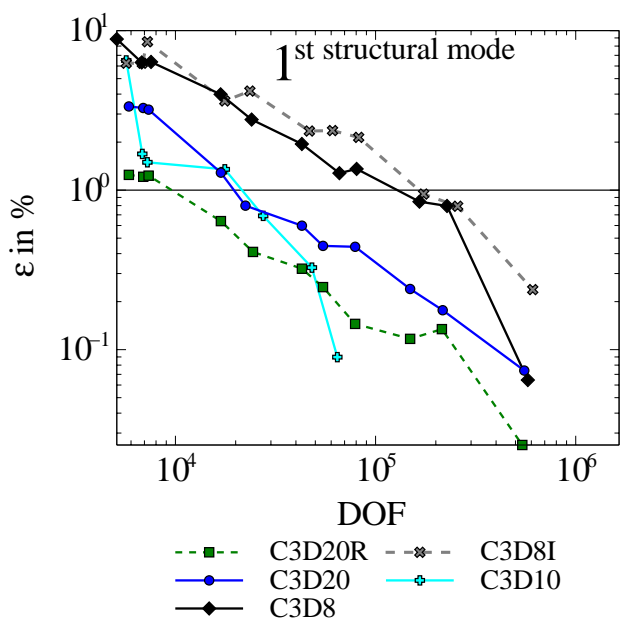

(b)

Fig. 15. Simplified crankcase results - first structural mode. (a) Eigenfrequency of first structural mode from finite element models with different types of elements and experiment and (b) relative error of first structural mode to a reference finite element model with $3.2 \cdot 10^{6} \mathrm{DOF}$ and quadratic elements.

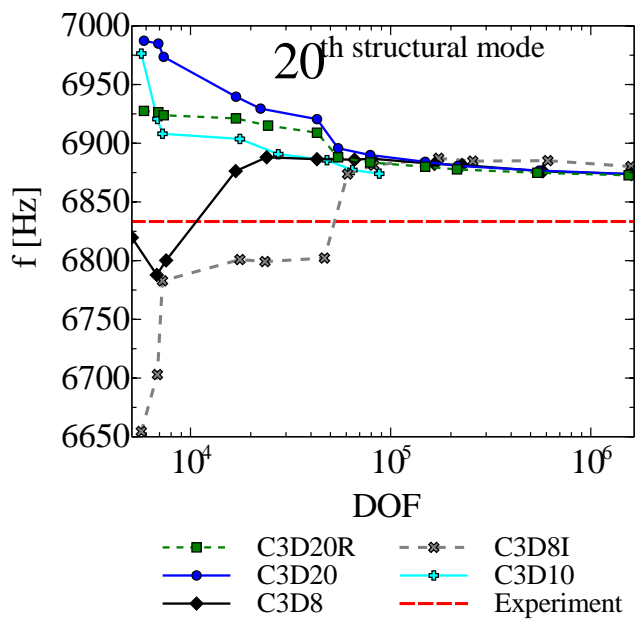

(a)

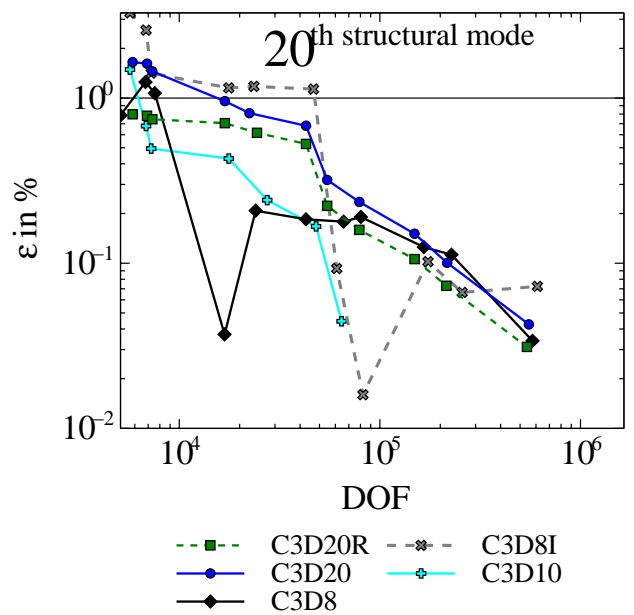

(b)

Fig. 16. Simplified crankcase results - twentieth structural mode. (a) Eigenfrequency of twentieth structural mode from finite element models with different types of elements and experiment and (b) relative error of twentieth structural mode to a reference finite element model with $3.2 \cdot 10^{6} \mathrm{DOF}$ and quadratic elements.

A mode shape of a complex structure, which is determined by the geometry, the parameters of the material model and the boundary conditions, has an unknown shape, the elements may deform in a complex way. Therefore, the elements can deform arbitrarily. Because of this complex behavior, a straightforward forecast of the elements' performance is not 


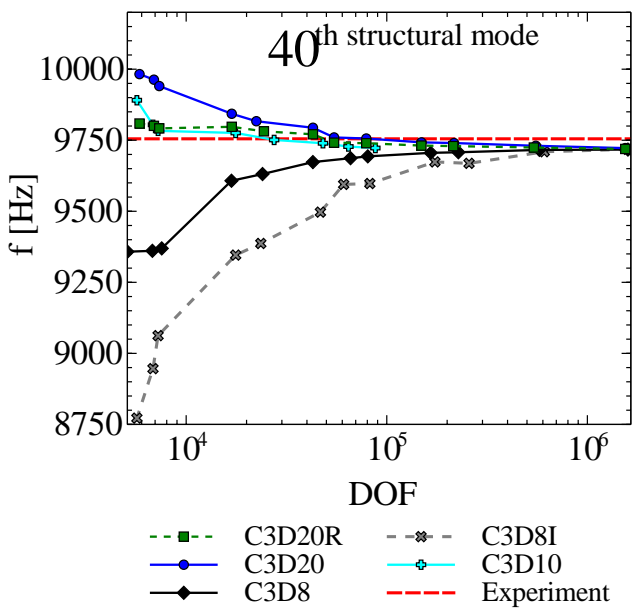

(a)

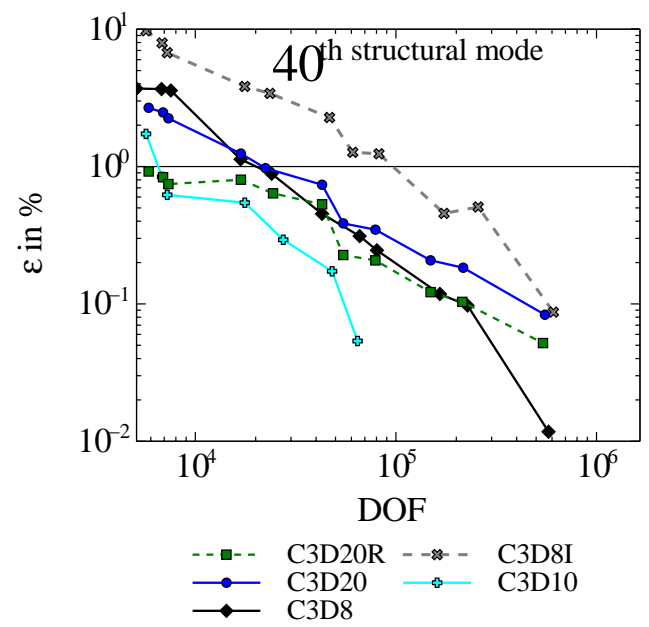

(b)

Fig. 17. Simplified crankcase results - fortieth structural mode. (a) Eigenfrequency of fortieth structural mode from finite element models with different types of elements and experiment and (b) relative error of fortieth structural mode to a reference finite element model with $3.2 \cdot 10^{6} \mathrm{DOF}$ and quadratic elements.

possible. The results displayed in Figs. 15, 17 are representative for the general elements' behavior. The reference model to calculate the relative error $\epsilon$ has $3.2 \cdot 10^{6}$ DOF. For the first structural mode, linear and quadratic elements converge from above. For the twentieth and fortieth structural modes, linear elements converge from below and quadratic elements from above. It can be seen that the converged solutions differ about $1 \%$ from the experimental results. This fact still holds for other and even higher-order modes, but the results

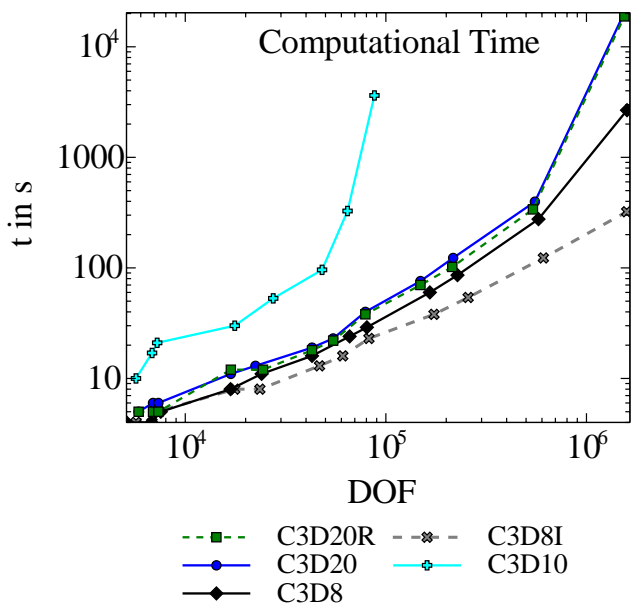

Fig. 18. Comparison of computational time (simplified crankcase) of the finite element models with different types of elements. 
are excluded due to the lack of new information. Quadratic elements perform better in terms of DOF expected for using linear elements C3D8. This means, in order to achieve a similar simulation quality, two orders of DOF less can be used when choosing quadratic brick elements instead of linear elements. Comparing quadratic hexahedral and quadratic tetrahedral elements with each other, it is possible to see that the performance is comparable. The only exception is the usage of the C3D8I elements. With the same number of DOF, the C3D8 models have more accuracy than C3D8I models. The latter elements tend to give the worst accuracy in terms of DOF for this model, but have the advantage of having the lowest CPU time. The models meshed with quadratic tetrahedral elements yield a more accurate solution for higher structural modes than quadratic hexahedral elements with the same number of DOF.

\subsection{Computational time in terms of DOF}

Taking into account the CPU time in Fig. 18 needed to obtain the results, it is obvious that the tetrahedral quadratic element type achieves more accurate results in less wall clock time. For these investigations, the standard eigensolver Lanczos was used.

\section{Conclusion}

The authors presented a finite element performance study based on the assumption that single element benchmark tests do not provide reliable information when choosing appropriate mesh properties for structural FE-analysis. Experimental tests set the basis to evaluate the simulations' quality with respect to the highest possible accuracy of converged solutions. Since the experimentally identified eigenfrequencies of simple structures vary within $\pm 2 \%$ due to material and geometry parameter uncertainties, it is not useful to define a numerical model with a accuracy below a deviation of $1 \%$. The provided guideline for choosing efficient discretization parameters for thin-walled structures helps the engineer to identify the element type and DOF for structural analysis in order to achieve a certain degree of accuracy with respect to experimental results. The rule of thumb for meshing thin-walled structures states that

(i) the user should use at least 20 quadratic or 500 linear elements per standing bending structural wave. Such a mesh gives a converged solution for eigenfrequencies of bending modes with an error of less than $1 \%$, which is considered as a reasonable compromise between the accuracy of an experiment and the variation of samples and their parameters,

(ii) when using linear elements, good conditioning of the element shape close to the cubic form is essential,

(iii) the pollution effect is not relevant for three-dimensional structured meshes in the frequency range up to medium to high frequencies when measuring the error based on eigenfrequencies. The investigations show a decreasing error for a constant number of elements per wavelength with increasing frequency, 
Table 4. Summarized pros and cons of the element types discussed in the paper; complex geometry: investigated crankcase; real complex geometry in industrial applications: geometry including sharp edges, beads, ribs, blind holes.

\begin{tabular}{|c|c|c|}
\hline Type & Pros & Cons \\
\hline C3D20 & $\begin{array}{l}\text { - Good conditioning of element shape } \\
\text { less essential } \\
\text { - Suitable for complex geometry }\end{array}$ & $\begin{array}{l}\text { - Needs a structured mesh } \\
\text { - Not suitable for real complex industrial } \\
\text { structures }\end{array}$ \\
\hline C3D20R & $\begin{array}{l}\text { - Highest accuracy with lowest num- } \\
\text { ber of DOF } \\
\text { - Suitable for complex geometry }\end{array}$ & $\begin{array}{l}\text { - Needs a structured mesh } \\
\text { - Not suitable for real complex industrial } \\
\text { structures }\end{array}$ \\
\hline C3D10 & $\begin{array}{l}\text { - Unstructured mesh possible } \\
\text { - Suitable for real complex industrial } \\
\text { - } \text { structures } \\
\text { - High accuracy with respect to DOF }\end{array}$ & \\
\hline C3D8 & - Suitable for complex geometry & $\begin{array}{l}\text { - Needs a structured mesh } \\
\text { - Not suitable for real complex industrial } \\
\text { structures } \\
\text { - Good conditioning of element shape } \\
\text { essential }\end{array}$ \\
\hline C3D8I & $\begin{array}{l}\text { - Most accurate linear element with } \\
\text { respect to DOF for regular mesh } \\
\text { - Suitable for complex geometry }\end{array}$ & $\begin{array}{l}\text { - Needs a structured and regular mesh } \\
\text { - Not suitable for real complex industrial } \\
\text { structures } \\
\text { - Good conditioning of element type } \\
\text { highly essential }\end{array}$ \\
\hline
\end{tabular}

(iv) quadratic elements perform much better than linear elements when comparing results of models with identical DOF and are therefore much more efficient and

(v) calculations using a regular mesh greatly benefit in performance.

A detailed list of advantages and disadvantages of each element type are summarized in Table 4. Corroborated by the results of this study, the authors recommend the use of quadratic elements as long as the user is able to determine that all field gradients are monotonic inside the element. In this case, the fact that quadratic elements are not unconditionally stable is uncritical. Similar conclusions are found in Ramesh et al ${ }^{46}$ and Marburg and Schneider ${ }^{17}$ for acoustic-wave propagation problems with respect to the efficiency of quadratic boundary elements. For the future, investigation of higher-order elements is planed. However, only a few commercial finite element codes offer this feature which is one reason why higher-order elements are hardly used in industry so far.

\section{Acknowledgments}

The authors acknowledge the financial support of the Bavarian Research Foundation. 


\section{References}

1. K.-J. Bathe, Finite Element Procedures (Prentice-Hall, New Jersey, NJ, USA, 1996).

2. F. Koschnick, Geometric locking phenomena of finite elements and a uniform concept for their elimination, Dissertation, Technische Universität München, Germany (2004).

3. J. Argyris and H.-P. Mlejnek, Die Methode der Finiten Elemente (Vieweg, Braunschweig, 1988).

4. N. Maia, J. Silva, N. Lieven, G. Skingle, W. To and A. Urgueira, Theoretical and Experimental Modal Analysis (Research Studies Press Ltd., Hertfordshire, 1998).

5. D. G. Crighton, A. P. Dowling, J. E. Ffowcs, M. Heckl and L. F. A., Modern Methods in Analytical Acoustics (Lecture Notes) (Springer-Verlag, London, 1992).

6. M. Kompella and R. Bernhard, Variation of structural-acoustic characteristics of automotive vehicles, Noise Control Eng. J. 44 (1996) 93-99.

7. E. Hills, B. R. Mace and N. S. Ferguson, Acoustic response variability in automotive vehicles, J. Sound Vib. 321 (2009) 286-304.

8. M. Hornikx, M. Kaltenbacher and S. Marburg, A platform for benchmark cases in computational acoustics, Acta Acust. United with Acust. 101 (2015) 811-820.

9. S. Marburg, H.-J. Beer, J. Gier, H.-J. Hardtke, R. Rennert and F. Perret, Experimental verification of structural acoustic modeling and design optimization, J. Sound Vib. 252 (2002) $591-615$.

10. A. Cifuentes and A. Kalbag, A performance study of tetrahedral and hexahedral elements in 3D finite element structural analysis, Finite Elem. Anal. Des. 12 (1992) 313-318.

11. N.-S. Lee and K.-J. Bathe, Effects of element distortions on the performance of isoparametric elements, Int. J. Numer. Methods Eng. 36(20) (1993) 3553-3576.

12. P. Langer, K. Sepahvand, M. Krause and S. Marburg, Simple vibroacoustic systems - Influence of uncertainties in simulation and experiment, in Proc. NOVEM 2015, ed. G. Pavic (Dubrovnik, Croatia, 2015).

13. I. Babuška, F. Ihlenburg, T. Paik and A. Sauter, A generalized finite element methode for solving the Helmholtz equation in two dimensions with minimal pollution, Comput. Methods Appl. Mech. Eng. 128 (1995) 325-359.

14. F. Ihlenburg, Finite Element Analysis of Acoustic Scattering (Springer-Verlag, New York, 1998).

15. L. L. Thompson and P. M. Pinsky, Complex wave number Fourier analysis of the $p$-version finite element method, Comput. Mech. 13 (1994) 255.

16. E. H. Ooi, H. Dogan and V. Popov, Dispersion analysis of the meshless local boundary integral equation and radial basis integral equation methods for the Helmholtz equation, J. Eng. Anal. Bound. Elem. 50 (2015) 360-371.

17. S. Marburg and S. Schneider, Influence of element types on numeric error for acoustic boundary elements, J. Comput. Acoust. 11 (2003) 363-386.

18. S. Marburg, Six boundary elements per wavelength: Is that enough? J. Comput. Acoust. 10 (2002) 25-51.

19. S. Marburg, The Burton and Miller method: Unlocking another mystery of its coupling parameter, J. Comput. Acoust. 24 (2015) 20.

20. M. Newman and A. Pipano, Fast modal extraction in NASTRAN via the FEER computer program, NASA. Langley Res. Center NASTRAN: Users Experiences (1973), pp. 485-506.

21. B. N. Parlett, The Symmetric Eigenvalue Problem (Prentice-Hall, New Jersey, NJ, USA, 1980).

22. J. H. Wilkinson, The Algebraic Eigenvalue Problem (Numerical Mathematics and Scientific Computation) (Oxford Science Publications, Oxford, 1999).

23. K.-J. Bathe and E. Wilson, Solution methods for eigenvalue problems in structural mechanics, Int. J. Numer. Methods Eng. 6 (1972) 213-226.

24. D. S. Simulia, ABAQUS V6.10 Documentation (2010). 
25. K. J. Bathe, Finite Element Procedures in Engineering Analysis (Prentice-Hall, New Jersey, NJ, 1982).

26. O. C. Zienkiewicz and R. L. Taylor, The Finite Elemente Methode Volume 1: Basic Formulation and Linear Problems (MacGraw-Hill Book Company, New York, 1989).

27. O. C. Zienkiewicz and R. L. Taylor, The Finite Element Method Volume 2: Solid and Fluid Mechanics, Dynamics and Non-Linearity (MacGraw-Hill Book Company, New York, 1991).

28. J. Barlow, Optimal stress locations in finite element models, Int. J. Numer. Methods Eng. 10(2) (1976) 243-251.

29. R. C. Averill and J. N. Reddy, Behaviour of plate elements based on the first-order shear deformation theory, Eng. Comput. 7 (1990) 57-74.

30. J. N. Reddy, On locking-free shear deformable beam finite elements, Comput. Methods Appl. Mechan. Eng. 149(1-4) (1997) 113-132.

31. G. Prathap and G. R. Bhashyam, Reduced integration and the shear flexible beam element, $J$. Numer. Methods Eng. 18 (1982) 195-210.

32. D. J. Ewins, Modal Testing: Theory and Practice (Research Studies Press, Letchworth, Hertfordshire, England, 1984).

33. International Organization for Standardization (ISO), Methods for the Experimental Determination of Mechanical Mobility; Parts 1-5 (Switzerland, 1986-1994).

34. I. Vibrant Technology, ME'scope Version 5.0 Operating Manual (2009).

35. P. Langer, K. Sepahvand and S. Marburg, Uncertainty quantification in analytical and finite element beam models using experimental data, in Proc. 9th Int. Conf. Structural Dynamics, EURODYN 2014, eds. A. Cunha, E. Caetano, P. Ribeiro and G. Müller (Porto, Portugal, 2014).

36. I. Babuška and S. A. Sauter, Is the pollution effect of the FEM avoidable for the Helmholtz equation considering high wave number? SIAM J. Numer. Anal. 34 (1997) 2392-2423.

37. F. Ihlenburg and I. Babuška, Finite element solution of the Helmholtz equation with high wavenumber Part I: The h-version of the FEM, Comput. Math. Appl. 30 (1995) 9-37.

38. F. Ihlenburg and I. Babuška, Finite element solution of the Helmholtz equation with high wavenumber Part II: The h-version of the FEM, SIAM J. Numer. Anal. 34 (1997) 315-358.

39. M. Ainsworth, Discrete dispersion relation for hp-version finite element approximation at high wave number, SIAM J. Numer. Anal. 42 (2004) 553-575.

40. I. Babuška, F. Ihlenburg, E. Paik and S. A. Sauter, A generalized finite element method for solving the Helmholtz equation in two dimensions with minimal pollution, Comput. Methods Appl. Mech. Eng. 128 (1995) 325-359.

41. S. Marburg, Normal modes in external acoustics. Part I: Investigation of the one-dimensional duct problem, Acta Acust. United Acust. 91 (1986) 1063-1078.

42. A. A. Oberai and P. M. Pinsky, A numerical comparison of finite element methods for the Helmholtz equation, J. Comput. Acoust. 8 (2000) 211-221.

43. E. Q. Sun, Shear locking and hourglassing in MSC Nastran, ABAQUS, and ANSYS, MSC.Software Virtual Product Development Conference (VPD 2006), Huntington, CA., July 17-19 (2006).

44. T. Belytschko, H. Stolarski, W. K. Liu, N. Carpenter and J. Ong, Stress projections for membrane and shear locking in shell finite elements, Comput. Methods Appl. Mech. Eng. 51(1-3) (1985) 221-258.

45. I. Babuška and M. Suri, On locking and robustness in the finite element method, Report BN1112, Inst. for Physical Sci. and Tech., University of Maryland, College Park Campus, May 1990.

46. S. S. Ramesh, K.-M. Lim and B. C. Khoo, Comparison of constant and discontinuous quadratic boundary elements for exterior axisymmetric acoustic-wave propagation problems, J. Comput. Acoust. 23 (2015) 22. 